\title{
Can Biofluids Metabolic Profiling Help to Improve Healthcare during Pregnancy?
}

\author{
Gonçalo Graça, ${ }^{1}$ Sílvia O. Diaz, ${ }^{1}$ Joana Pinto, ${ }^{1}$ António S. Barros, ${ }^{2}$ Iola F. Duarte, ${ }^{1}$ \\ Brian J. Goodfellow, ${ }^{1}$ Eulália Galhano, ${ }^{3}$ Cristina Pita, ${ }^{3}$ Maria do Céu Almeida, ${ }^{3}$ \\ Isabel M. Carreira, ${ }^{4}$ and Ana M. Gil ${ }^{1}$ \\ ${ }^{1}$ CICECO Department of Chemistry, University Campus of Santiago, University of Aveiro, \\ 3810-193 Aveiro, Portugal \\ ${ }^{2}$ QOPNA Research Unit, Department of Chemistry, University Campus of Santiago, \\ University of Aveiro, 3810-193 Aveiro, Portugal \\ ${ }^{3}$ Maternidade Bissaya Barreto, Centro Hospitalar de Coimbra, 3000 Coimbra, Portugal \\ ${ }^{4}$ Cytogenetics and Genomics Laboratory, Faculty of Medicine, University of Coimbra and \\ Forensic Science Centre (CENCIFOR), Coimbra, Portugal
}

Correspondence should be addressed to Ana M. Gil, agil@ua.pt

Copyright (C) 2012 Gonçalo Graça et al. This is an open access article distributed under the Creative Commons Attribution License, which permits unrestricted use, distribution, and reproduction in any medium, provided the original work is properly cited.

\begin{abstract}
This paper describes a metabonomics study of 2nd trimester biofluids (amniotic fluid, maternal urine, and blood plasma), in an attempt to correlate biofluid metabolic changes with suspected/diagnosed fetal malformations (FM) and chromosomal disorders as well as with later occurring gestational diabetes mellitus (GDM), preterm delivery (PTD), and premature rupture of membranes (PROM). The global biochemical picture given by the threesome of biofluids should enable the definition of potential disease signatures and unveil potential metabolite markers for clinical use in predictive prenatal diagnostics. Results show that relatively strong metabolic disturbances accompany FM, reflected in all three biofluids and thus suggesting the involvement of both fetal and maternal metabolisms. Regarding GDM, amniotic fluid and maternal urine seem potential good media to detect early metabolic changes, and PTD subjects show small metabolite changes in the same biofluids, undergoing work being focused on plasma composition. Chromosomal disorders show an interestingly marked effect on maternal urine, whereas no statistically relevant early changes have been observed for PROM subjects. Interestingly, in the case of FM and chromosomal disorders, maternal biofluids show some sensitivity to disorder type, for example, for central nervous system malformations and trisomy 21, respectively. These results show the usefulness of biofluid metabonomics to probe overall metabolic disturbances in relation to prenatal disorders.
\end{abstract}

Keywords: Prenatal health, pregnancy, diagnosis, diabetes, preterm, trisomy, amniotic fluid, urine blood, metabolomics, metabonomics, NMR, multivariate analysis

\section{Introduction}

Prenatal diagnostic methods include a variety of procedures, with emphasis on routine ultrasound testing, often in combination with maternal serum markers, for detection of chromosomal disorders 
and fetal malformations. For higher risk pregnancies, diagnosis of chromosomal anomalies or specific genetic diseases is based on chorionic villus sampling, amniocentesis and cordocentesis, invasive procedures which still carry some risk of miscarriage, infection, or premature birth [1]. In addition, disorders such as gestational diabetes mellitus (GDM), preeclampsia (PE), preterm delivery (PTD), small for gestational age (SGA), or intrauterine growth restriction (IUGR) may pose a significant risk to mother and fetus but cannot easily be predicted by the presently available methods.

This paper describes the use of metabonomics, mainly based on nuclear magnetic resonance (NMR) spectroscopy, as a holistic analytical strategy in the quest for new and earlier biomarkers of both fetal and maternal health. In this context, previous reports of NMR of amniotic fluid (AF) have addressed, at a preliminary level, fetal maturity [2,3], gestational age [4, 5], and several disorders, for example, PE, fetal malformations (FM), GDM, PTD, and premature rupture of membranes (PROM) [2, 6-8]. Metabolite profiling of urine and blood has been, however, less developed, in spite of the great potential of these biofluids for less-invasive diagnosis methods. In this context, some studies on blood have focused on PE, employing both NMR $[9,10]$ and ultra high performance liquid chromatography and mass spectrometry (UPLC-MS) [11]. Hereby, a metabonomics study of 2nd trimester biofluids (AF, maternal urine, and blood plasma) is presented, in an attempt to correlate biofluid metabolic changes with suspected/diagnosed FM and chromosomal disorders, and with GDM, PTD, and PROM developed later in pregnancy. The global biochemical picture given by the threesome of biofluids should enable the definition of potential disease signatures and unveil potential metabolite markers for clinical use in prenatal diagnostics and prognostics.

\section{Experimental}

\subsection{Samples}

Amniotic fluid (AF), urine, and blood plasma samples were collected at the time of amniocentesis (1425 gestational weeks, g.w.), of pregnant women aged $>35$ or based on medical history, and stored at $-80^{\circ} \mathrm{C}$ until analysis. All pregnancies were followed until birth, eventually defining sample groups according to their clinical outcome: controls, FM, prediagnostic GDM (for women diagnosed with GDM later in their pregnancy), pre-PTD (for women who gave birth prior to 37 g.w.), pre-PROM (for women who had prelabor rupture of membranes after 37 g.w.), and chromosomal disorders (generally diagnosed ca. 2 weeks after amniocentesis). Other disorders were not considered at this stage due to insufficient sample numbers. Table 1 lists sample numbers, and detailed sample preparation for analysis is described elsewhere $[8,12]$.

\subsection{NMR Spectroscopy}

All 1D and 2D NMR spectra were recorded on a Bruker Avance DRX 500 spectrometer equipped with an actively shielded gradient unit with a maximum gradient strength output of $53.5 \mathrm{G} / \mathrm{cm}$, at $300 \mathrm{~K}$. The detailed description of the acquisition conditions and parameters employed is described elsewhere $[8,12]$. 
Table 1: List of 2nd trimester amniotic fluid, urine, and blood plasma samples collected.

\begin{tabular}{lccc}
\hline Condition & AF & Urine & Blood plasma \\
\hline Controls* $^{*}$ & 82 & 25 & 20 \\
Fetal malformations (FM) & 27 & 29 & 27 \\
Prediagnostic gestational diabetes mellitus & 27 & 29 & 14 \\
(GDM)* $^{*}$ & 12 & 19 & 6 \\
Preterm delivery (PTD)* & 5 & 5 & 2 \\
Preeclampsia (PE) & 34 & 38 & 18 \\
Premature rupture of membranes (PROM)* & 6 & 5 & 4 \\
Intrauterine growth restriction (IUGR) $_{\text {Chromosomal disorders (CD) }}^{*}$ & $10(1)$ & $25(9)$ & $23(8)$ \\
\hline
\end{tabular}

* Groups with sufficient sample numbers to allow multivariate analysis to be performed, at this stage. Numbers in brackets indicate number of trisomy 21 cases.

\subsection{Chemometrics}

NMR data were processed for chemometrics using normalization to total spectral area and different variable scaling methods for comparison purposes (unit variance, Pareto and centered scaling). Principal component analysis (PCA) [13] and partial least squares discriminant analysis (PLS-DA) [14] and its orthogonal variant (O-PLS-DA) [15] were applied to the data, using SIMCA-P 11.5 (Umetrics, Umeå, Sweden). Monte-Carlo cross-validation (MCCV) was also applied, along with spectral integration for relevant signals.

\section{Results and Discussion}

Given the sample numbers shown in Table 1, only the results obtained for the highlighted groups are presented below. Figure 1 shows typical 1D standard ${ }^{1} \mathrm{H}$ NMR spectra for 2 nd trimester AF and maternal blood plasma and urine, illustrating the complex nature of the data, which comprises many tens hundreds of signals, greatly overlapped in some spectral regions. As expected, it is clearly seen that the plasma ${ }^{1} \mathrm{H}$ NMR spectrum is dominated by broader lipid resonances, whereas both AF and urine spectra show a predominance of narrow resonances. In the case of AF and plasma, a set of additional 1D experiments has been carried out (not shown) in an attempt to reveal further spectral information: the Carr-PurcellMeiboom-Gill (CPMG) experiment which records only the narrower signals from fast tumbling, that is, smaller metabolites, and a diffusion-edited experiment which records the broad profile of slower tumbling molecules such as proteins and lipids. The wealth of compositional information present in ${ }^{1} \mathrm{H}$ NMR spectra has been translated into a list of metabolites present in each biofluid, through a range of $2 \mathrm{D}$ NMR experiments (homonuclear and heteronuclear correlations and $J$-resolved) [16] and comparison of spectral profiles with those found in spectral databases (Bruker Biorefcode database and other databases available online [17, 18]).

In this way, a metabolite profile has been established for each biofluid $[7,8,12]$ as typical of 2nd trimester controls (healthy pregnancies throughout) and serving as a reference to the detection of eventual spectral profile changes related to each disorder, in order to identify statistically relevant 


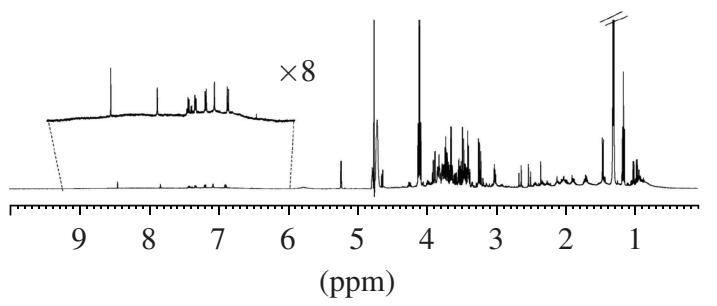

(a)

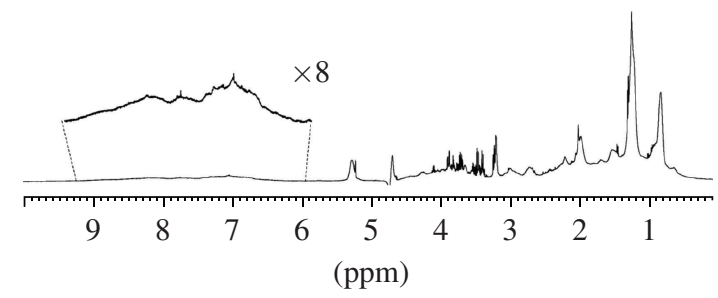

(b)

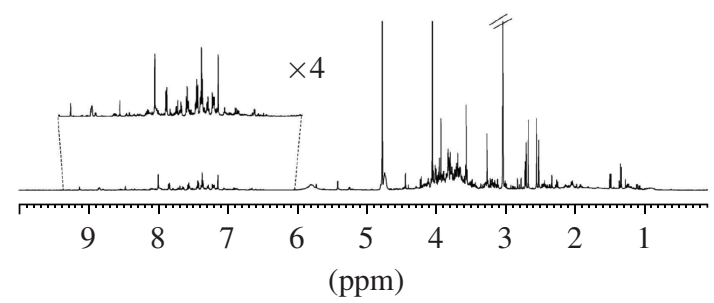

(c)

Figure 1: Typical standard ${ }^{1} \mathrm{H}$ NMR spectra of 2nd trimester (a) amniotic fluid, (b) maternal blood plasma, and (c) maternal urine.

disorder signatures. This has been carried out with the aid of multivariate analysis of the ${ }^{1} \mathrm{H}$ NMR 1D spectra, and Figure 2(a) shows a PLS-DA scores plot of the ${ }^{1} \mathrm{H}$ NMR spectra of amniotic fluid corresponding to controls and FM groups. The apparent group separation suggests that some differences exist between the spectral profiles representative of the two groups; however, this must be evaluated and interpreted with care, employing both MCCV validation and confirmation of spectral changes through spectral inspection and, when possible, signal integration and univariate analysis $(P$ value $<0.05)$. In the case shown, the statistical relevance of the group separation observed is expressed by a sufficiently high $Q^{2}$ value (0.38) and classification rate (89\%), as given by MCCV validation [8]. Interpretation of the underlying origins of group separation was based on the corresponding loadings plot (Figure 2(b)), which shows positive and negative peaks, corresponding to metabolites more concentrated in controls and in the FM group, respectively. In this way, changed levels were noted in 23 metabolites present in the AF of malformed fetuses: increased ascorbate, creatinine, glycoproteins, glycine, glutamine, lactate, methionine, serine, succinate, and threonine along with decreased $\alpha$-oxoisovalerate, alanine, phenylalanine, glucose, isoleucine, leucine, pyruvate, tyrosine, valine, and 4 unknowns [8]. Additional UPLC-MS work on amniotic fluid [19] has detected increases in carnitine, pyroglutamate, and polyol contents. The metabolite changes noted are consistent with enhanced glycolysis, possibly under fetal 


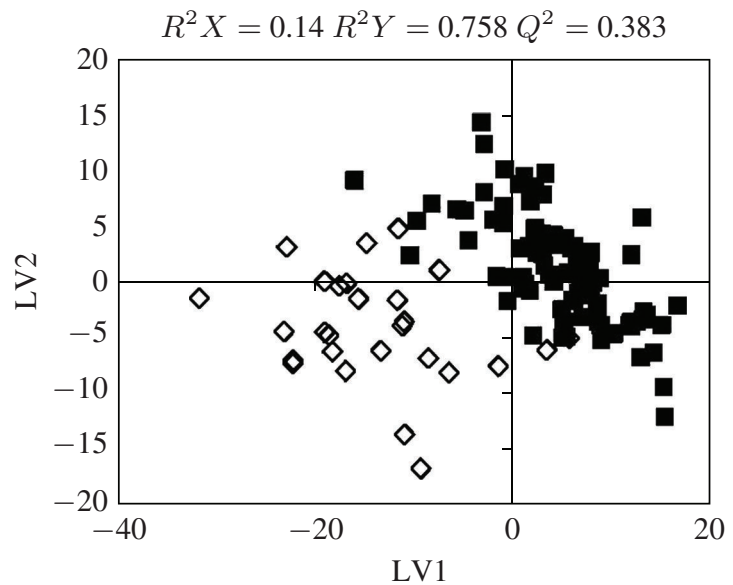

(a)

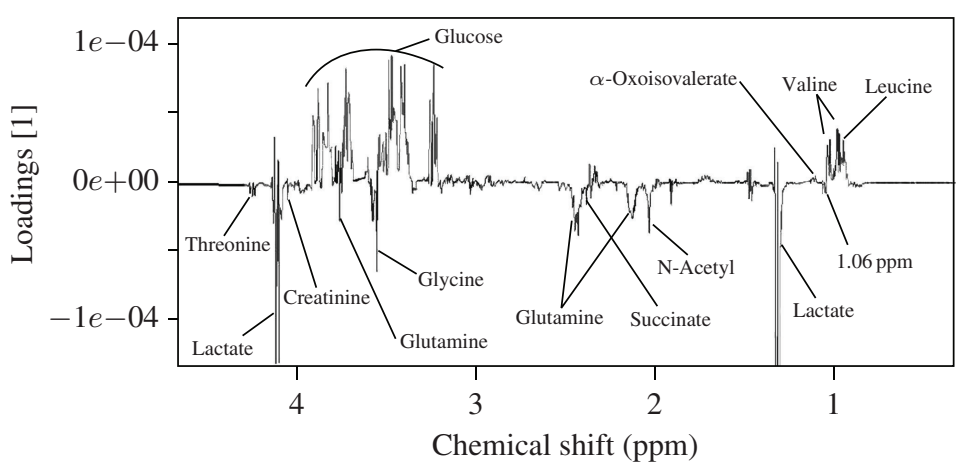

(b)

Figure 2: PLS-DA scores and LV1 loadings for NMR of AF for controls and FM groups.

hypoxia (originating enhanced usage of glucose and lactate increase) and reduced use of the respiratory chain pathway (consistent with a succinate increase). In relation to amino acids, the decreases in glucogenic amino acids (alanine, isoleucine, glutamate, methionine, phenylalanine, tyrosine, valine) may indicate their enhanced use in gluconeogenesis for the replenishment of glucose, whereas increases in glycine, glutamine, serine, and threonine suggest the possible involvement of these amino acids in other pathways. For instance, a higher glutamine level suggests fetal kidney underdevelopment, which is also consistent with the observed accumulation of glycoproteins. Glycine and serine increases suggest a disturbance in folic acid pool regulation, with possible reflections in amino acid and choline metabolisms. Other indicators relate to the marked decreases in leucine and $\alpha$-oxoisovalerate, which may indicate a higher demand for protein synthesis in malformed fetuses. Finally, the marked increase in ascorbate may also reflect amino acid and/or nucleotide sugar biosynthesis alterations since this compound is involved in sugar interconversion pathways. These metabolic disturbances have been supported by the parallel analysis of maternal plasma and urine [12], which has provided further evidence for hypoxia and enhanced lipid oxidation, together with choline metabolism disturbance. In addition, preliminary results on plasma suggest a metabolic-based separation of central nervous system 


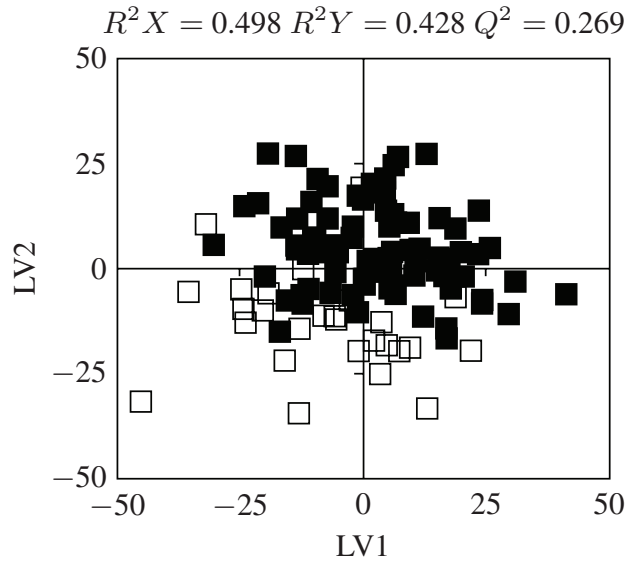

(a)

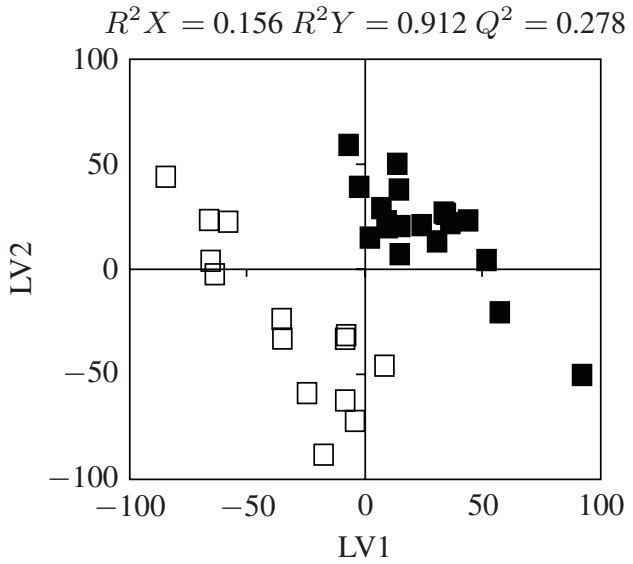

(b)

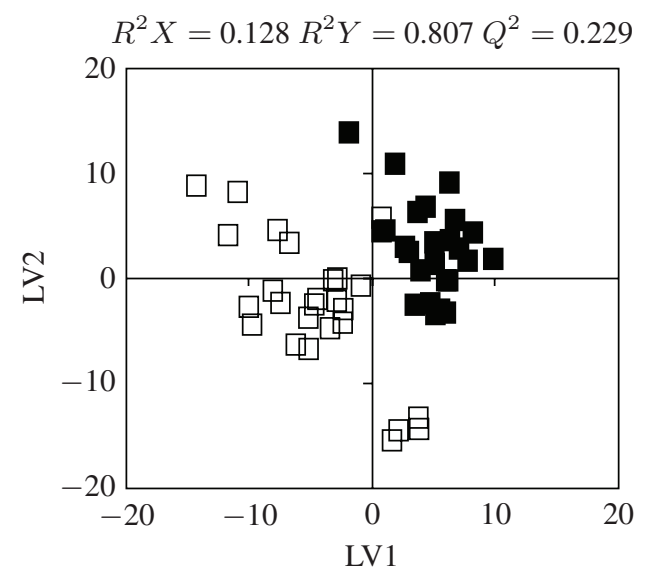

(c)

Figure 3: Comparison of PLS-DA scores obtained for (a) amniotic fluid, (b) blood plasma, and (c) urine corresponding to control and prediagnostic GDM groups.

malformation cases from the remaining types (cardiac, urogenital, soft tissues, and pulmonary), thus opening interesting possibilities as to the specificity of different biofluids for different malformation types.

A similar strategy has been applied to the remaining disorder groups considered in this study: prediagnostic GDM, pre-PTD, pre-PROM, and chromosomal disorders. The PLS-DA score plots obtained for each of the three biofluids corresponding to control and prediagnostic GDM groups reflect a weaker metabolic impact of this disorders, expressed by the lower $Q^{2}$ values (Figure 3). Interestingly, the results have shown that a small increase in glucose is apparent in AF [8], but not in the corresponding plasma and urine, at the time of sample collection, that is, several weeks before clinical diagnosis [12]. In addition, changes in 9 further metabolites have been noted in AF (creatinine, acetate, formate, glutamine, glycine, proline, glycerophosphocholine (GPC), serine, and taurine) along with betaine and trimethylamine- $\mathrm{N}$-oxide in blood and 7 metabolites in urine (3-hydroxyisovalerate, 
Table 2: Summary of the impact of disorders on the metabolite profiling of 2nd trimester amniotic fluid, urine, and blood plasma.

\begin{tabular}{lccccc}
\hline Disorder & FM & Prediagnostic GDM & Pre-PTD & Pre-PROM & Chromosomal disorders \\
\hline Biofluid & & & & & \\
Amniotic fluid & +++ & ++ & + & + & + \\
Maternal blood plasma & $+++{ }^{*}$ & + & a & b & $++^{* *}$ \\
Maternal urine & +++ & ++ & + & b & $++^{* *}$ \\
\hline
\end{tabular}

${ }^{\mathrm{a}}$ Insufficient sample numbers at this stage, ${ }^{\mathrm{b}}$ no metabolite changes noted, ${ }^{*}$ different malformation types, and ${ }^{* *}$ chromosomal disorders are apparently distinguishable, + small impact, ++ medium impact, +++ large impact.

2-hydroxyisobutyrate, choline, N-methyl-2-pyridone-5-carboxamide (2PY), N-methyl-nicotinamide (NMND), and 2 unknowns). In spite of the low magnitude of these changes and the low predictive value of the models obtained so far $[8,12]$, small metabolic variations seem to characterise women who will develop GDM later on in pregnancy, particularly at the levels of fetal insulin management and nucleotide and amino acid metabolisms.

In relation to pre-PTD subjects (results not shown), allantoin (possible marker of oxidative stress) has been found to be raised in AF along with 4 decreased metabolites (alanine, citrate, myoinositol, and 1 unknown) [8]. In addition, maternal urine shows raised levels of 3 metabolites (2hydroxyisobutyrate, choline, and 1 unknown), suggesting early disturbances in choline and amino acid/gut microflora metabolisms, although not excluding a possible relationship to BMI values [12]. In relation to chromosomal disorders, it was interesting to note that lipids seem to play an important role as a reflection of metabolic disturbances, as viewed through blood lipoprotein profiles and a urine choline increase. Furthermore, it became apparent (results not shown) that the plasma lipid profiles may be sensitive to chromosomal disorder type, with trisomy 21 samples having been distinguished from other disorders [12]. Finally, pre-PROM subjects have shown no significant changes in biofluid composition, with the exception of a faint suggestion of amino acid metabolism disturbance, as viewed through AF [8].

\section{Conclusion}

Table 2 summarises the results described above, indicating relatively strong metabolic disturbances accompanying fetal malformations, reflected in all three biofluids (AF, maternal urine, and plasma) and thus suggesting the involvement of both fetal and maternal metabolisms. Regarding GDM, AF, and maternal urine seem to be potentially good media to detect small early metabolic changes that take place prior to the clinical diagnosis of increased blood glucose. Pre-PTD subjects show small metabolite changes in the same biofluids, undergoing work being focused on plasma composition. Chromosomal disorders show an interestingly marked effect on maternal urine, along with weaker variations noted in $\mathrm{AF}$ and blood plasma. In the case of heterogeneous groups such as FM and chromosomal disorders, the maternal biofluids have shown some sensitivity to disorder type, for example, for central nervous system malformations and trisomy 21 , respectively. Finally, no statistically relevant changes have been 
observed for pre-PROM subjects, although a suggestion of amino acid disturbance has been registered for amniotic fluid.

These results show the usefulness of biofluid metabonomics to probe overall metabolic disturbances in relation to prenatal disorders. This work may be pursued along several avenues, one involving the use of more sophisticated multivariate analysis methods to find concomitant and interconnected metabolite changes, either between datasets (e.g., NMR and MS) or between different biofluids. Furthermore, new developments should include a comprehensive follow-up study throughout the whole pregnancy period, so that time course information may be obtained and a more complete metabolic picture may be formed of pregnancy itself and the relevant disorders affecting it.

\section{Acknowledgments}

Funding is acknowledged from the European Regional Development Fund through the Competitive Factors Thematic Operational Programme and from the Foundation for Science and Technology (FCT), Portugal (research Project PTDC/QUI/66523/2006 and research Grants SFRH/BD/41869/2007 and SFRH/BD/64159/2009). The Portuguese National NMR Network (RNRMN), supported with FCT funds is also acknowledged, and the authors are grateful to M. Spraul, Bruker BioSpin, Germany, for providing access to spectral NMR databases.

\section{References}

[1] N. F. Hacker, J. G. Moore, and J. C. Gambone, Essentials of Obstetrics and Gynecology, Elsevier, New York, NY, USA, 4th edition, 2004.

[2] J. L. Bock, "Metabolic profiling of amniotic fluid by proton nuclear magnetic resonance spectroscopy: correlation with fetal maturation and other clinical variables," Clinical Chemistry, vol. 40, no. 1, pp. 56-61, 1994.

[3] B. N. Joe, K. Vahidi, A. Zektzer et al., "1H HR-MAS spectroscopy for quantitative measurement of choline concentration in amniotic fluid as a marker of fetal lung maturity: interand intraobserver reproducibility study," Journal of Magnetic Resonance Imaging, vol. 28, no. 6, pp. 1540-1545, 2008.

[4] C. J. Sims, D. T. Fujito, D. R. Burholt, J. Dadok, and D. Allan Wilkinson, "Comparison of metabolite levels in second and third trimester human amniotic fluid samples using proton magnetic resonance spectroscopy," Journal of Maternal-Fetal Investigation, vol. 6, no. 2, pp. 62-66, 1996.

[5] B. R. Cohn, E. Y. Fukuchi, B. N. Joe et al., "Calculation of gestational age in late second and third trimesters by ex vivo magnetic resonance spectroscopy of amniotic fluid," American Journal of Obstetrics and Gynecology, vol. 203, no. 1, pp. 76.e1-76.e10, 2010.

[6] P. M. W. Groenen, U. F. Engelke, R. A. Wevers et al., "High-resolution 1H NMR spectroscopy of amniotic fluids from spina bifida fetuses and controls," European Journal of Obstetrics Gynecology and Reproductive Biology, vol. 112, no. 1, pp. 16-23, 2004.

[7] G. Graça, I. F. Duarte, A. S. Barros et al., "1H NMR based metabonomics of human amniotic fluid for the metabolic characterization of fetus malformations," Journal of Proteome Research, vol. 8, no. 8, pp. 4144-4150, 2009.

[8] G. Graça, I. F. Duarte, A. S. Barros et al., "Impact of prenatal disorders on the metabolic profile of second trimester amniotic fluid: a nuclear magnetic resonance metabonomic study," Journal of Proteome Research, vol. 9, no. 11, pp. 6016-6024, 2010. 
[9] E. Turner, J. A. Brewster, N. A. B. Simpson, J. J. Walker, and J. Fisher, "Plasma from women with preeclampsia has a low lipid and ketone body content-a nuclear magnetic resonance study," Hypertension in Pregnancy, vol. 26, no. 3, pp. 329-342, 2007.

[10] E. Turner, J. A. Brewster, N. A. B. Simpson, J. J. Walker, and J. Fisher, "Aromatic amino acid biomarkers of preeclampsia—a nuclear magnetic resonance investigation," Hypertension in Pregnancy, vol. 27, no. 3, pp. 225-235, 2008.

[11] L. C. Kenny, D. I. Broadhurst, W. Dunn et al., "Robust early pregnancy prediction of later preeclampsia using metabolomic biomarkers," Hypertension, vol. 56, no. 4, pp. 741-749, 2010.

[12] S. O. Diaz, J. Pinto, G. Graça et al., "Metabolic biomarkers of prenatal disorders: an exploratory NMR metabonomics study of second trimester maternal urine and blood plasma," Journal of Proteome Research, vol. 10, no. 8, pp. 3732-3742, 2011.

[13] I. T. Jolliffe, Principal Component Analysis, Springer, New York, NY, USA, 2nd edition, 2002.

[14] M. Barker and W. Rayens, "Partial least squares for discrimination," Journal of Chemometrics, vol. 17, no. 3, pp. 166-173, 2003.

[15] J. Trygg and S. Wold, "Orthogonal projections to latent structures (O-PLS)," Journal of Chemometrics, vol. 16, no. 3, pp. 119-128, 2002.

[16] J. C. Lindon, J. K. Nicholson, E. Holmes, and J. R. Everett, "Metabonomics: metabolic processes studied by NMR spectroscopy of biofluids," Concepts in Magnetic Resonance, vol. 12, no. 5, pp. 289-320, 2000.

[17] D. S. Wishart, D. Tzur, C. Knox et al., "HMDB: the human metabolome database," Nucleic Acids Research, vol. 35, supplement 1, pp. D521-D526, 2007.

[18] E. L. Ulrich, H. Akutsu, J. F. Doreleijers et al., "BioMagResBank," Nucleic Acids Research, vol. 36, supplement 1, pp. D402-D408, 2008.

[19] G. Graça, B. J. Goodfellow, A. S. Barros et al., "UPLC-MS metabolic profiling of second trimester amniotic fluid and maternal urine and comparison with NMR spectral profiling for the identification of pregnancy disorder biomarkers," Molecular BioSystems, vol. 8, no. 4, pp. 1243-1254, 2012. 


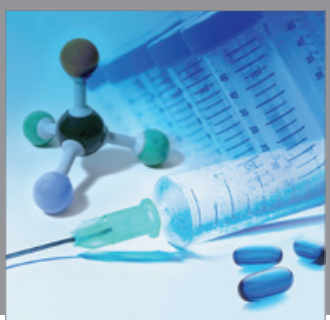

International Journal of

Medicinal Chemistry

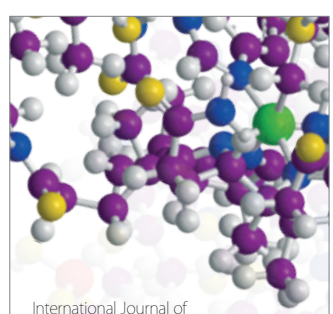

Carbohydrate Chemistry

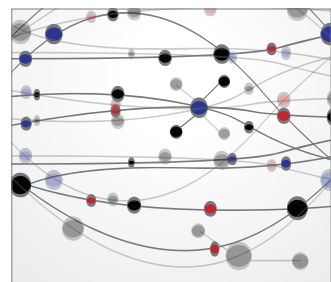

The Scientific World Journal
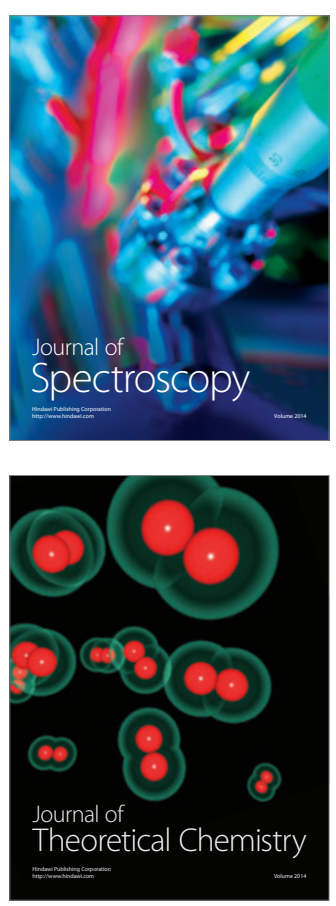
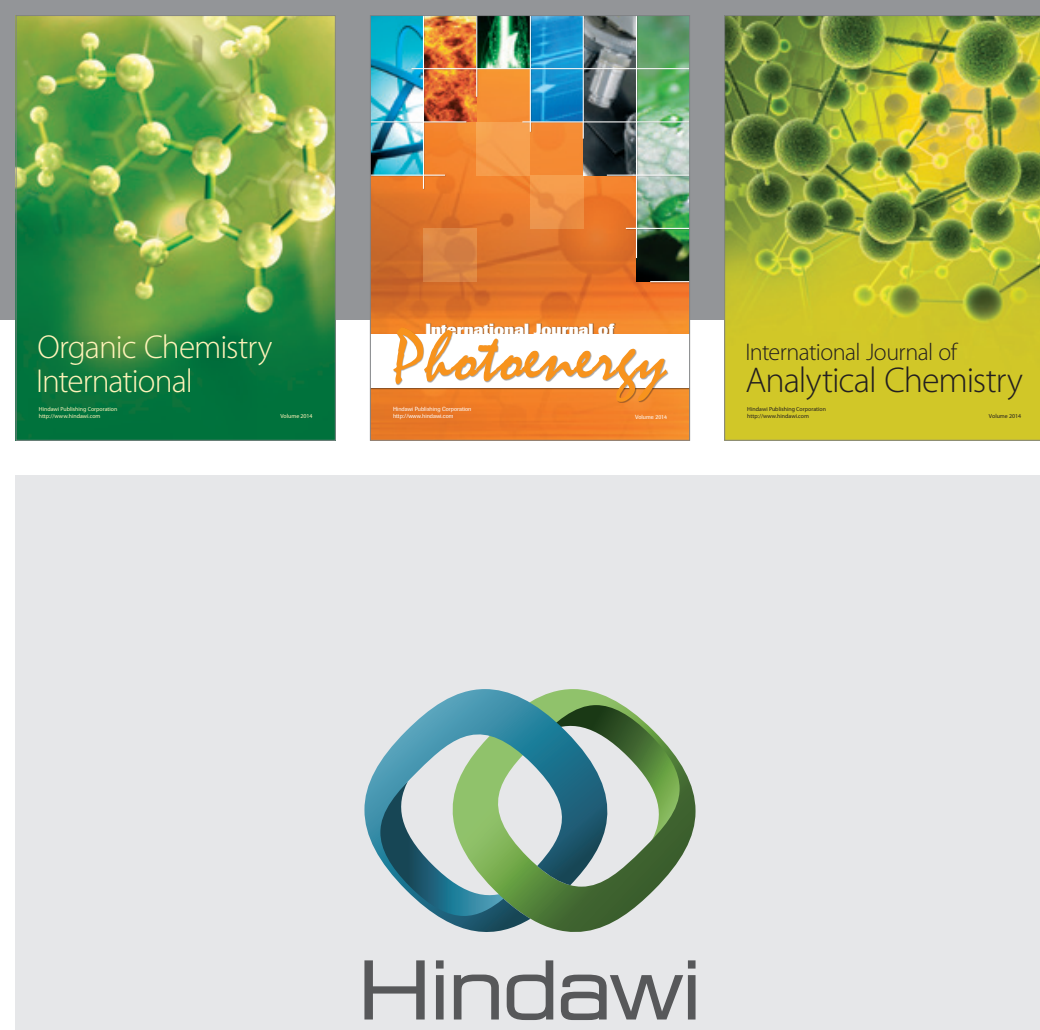

Submit your manuscripts at

http://www.hindawi.com
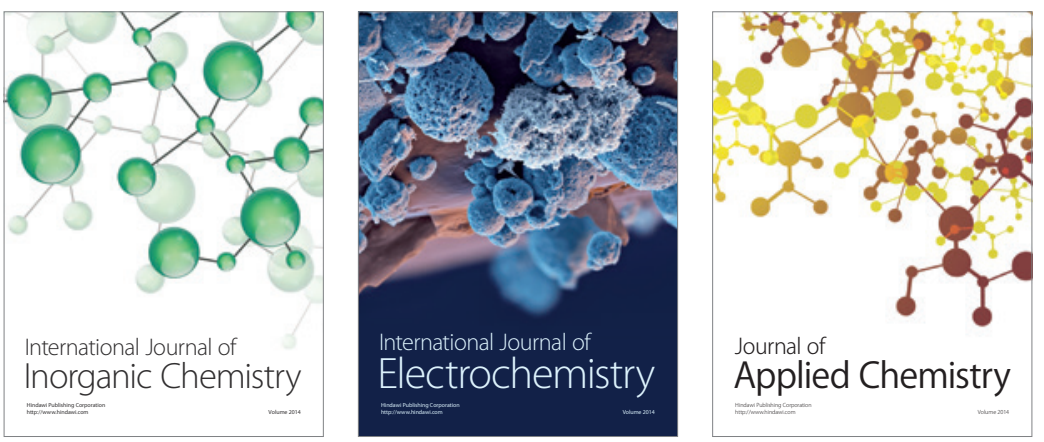

Journal of

Applied Chemistry
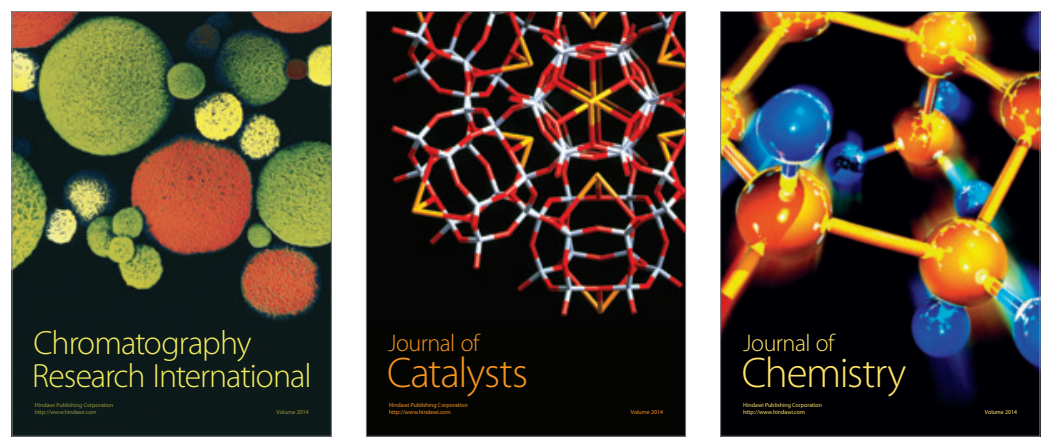
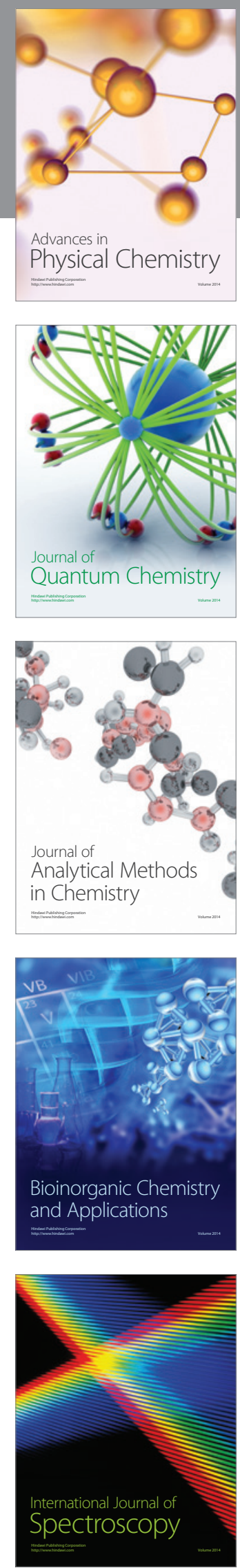